\title{
Global Comprehensive Literature Review and Meta-Analysis of Brucella spp. in Swine Based on Publications From 2000 to 2020
}

\author{
Qing-Long Gong 1,2, Yu-Han Sun ${ }^{1}$, Yang Yang ${ }^{1}$, Bo Zhao ${ }^{2}$, Qi Wang ${ }^{2}$, Jian-Ming Li ${ }^{1}$, \\ Gui-Yang Ge ${ }^{2}$, Zi-Yang Chen ${ }^{2}$, Kun Shi ${ }^{1}$, Xue Leng ${ }^{1}$, Ying Zong ${ }^{1 *}$ and Rui Du ${ }^{1,3,4 *}$ \\ ${ }^{1}$ College of Chinese Medicine Materials, Jilin Agricultural University, Changchun, China, ${ }^{2}$ College of Animal Science and \\ Technology, Jilin Agricultural University, Changchun, China, ${ }^{3}$ Laboratory of Production and Product Application of Sika Deer \\ of Jilin Province, Jilin Agricultural University, Changchun, China, ${ }^{4}$ Key Lab of Animal Production, Product Quality and \\ Security, Ministry of Education, Jilin Agricultural University, Changchun, China
}

OPEN ACCESS

Edited by:

Van Giap Nguyen,

Vietnam National University of Agriculture, Vietnam

Reviewed by: Faham Khamesipour, Shahid Beheshti University of Medical

Sciences, Iran

Dongbo Sun, Heilongjiang Bayi Agricultural

University, China

*Correspondence:

Ying Zong

zongying@jlau.edu.cn

Rui Du

durui197101@sina.com

Specialty section: This article was submitted to Veterinary Infectious Diseases,

a section of the journal

Frontiers in Veterinary Science

Received: 18 November 2020 Accepted: 06 April 2021 Published: 07 May 2021

Citation: Gong $Q-L$, Sun $Y-H$, Yang $Y$, Zhao $B$, Wang $Q$, Li J-M, Ge G-Y, Chen Z-Y, Shi $K$, Leng $X$, Zong $Y$ and $D u R$

(2021) Global Comprehensive Literature Review and Meta-Analysis of Brucella spp. in Swine Based on Publications From 2000 to 2020 Front. Vet. Sci. 8:630960 doi: 10.3389/fvets.2021.630960
Background: Brucellosis, a zoonotic disease, infects various hosts, including swine and humans. It has reemerged in recent years as a public health concern, and current studies on brucellosis infection in swine have been conducted worldwide. However, no meta-analyses of global brucellosis infection in swine have been published. The aim of this study was to provide an overview of Brucella species (spp.) in swine worldwide and the factors associated with its persistence.

Results: We searched seven databases for published epidemiological studies on brucellosis in pigs, including the Chinese National Knowledge Infrastructure, Wanfang Data, SpringerLink, ScienceDirect, Web of Science, the VIP Chinese Journal Database and PubMed. We selected 119 articles published from January 1, 2000 to January 3, 2020 for inclusion in the meta-analysis and analyzed the data using a random-effects model. Funnel plots and Egger's test showed significant publication bias in the included studies. The results of the sensitivity analysis showed that our study was relatively stable and reliable. The prevalence of brucellosis in swine was 2.1\% (95\% Cl: 1.6-2.6), of which the highest infection rate, which was found in Europe, was $17.4 \%$ (95\% Cl: 11.1-24.9). The prevalence in feral pigs $(15.0 \%, 95 \% \mathrm{Cl}$ : 8.4-23.2) was higher than that in domestic pigs (1.1\%, 95\% Cl 0.2-2.5). The prevalence in high-income countries (15.7\%, 95\% $\mathrm{Cl} 8.0-25.3)$ was significantly higher than that in middle- $(0.8 \%, 95 \% \mathrm{Cl} 0.5-1.1)$, and low-income countries $(0.1 \%, 95 \% \mathrm{Cl} 0.0-0.2)$. The prevalence was highest in finishing pigs at 4.9\% (95\% Cl 0.9-11.0), and lowest among suckling pigs at $0 \%(95 \% \mathrm{Cl} 0.0-0.5)$.

Conclusion: The Brucella prevalence in pig herds currently is distributed widely throughout the world. In some countries, swine brucellosis may be a neglected zoonotic disease. We recommend long-term monitoring of the prevalence of brucellosis in domestic and wild pig herds. Attention should also be paid to animal welfare on intensive pig farms; controlling the breeding density may play an important role in reducing the spread of brucellosis among pigs.

Keywords: brucellosis, brucella suis, meta-analysis, prevalence, swine 


\section{INTRODUCTION}

Brucellosis is a serious zoonotic disease caused by the Brucella species (spp), which occurs worldwide, especially in developing countries $(1,2)$. Although some developed countries have achieved freedom from animal brucellosis, it has reemerged in Japan, Australia and some European countries (Germany, Finland, Austria, Belgium and Italy) during the past 3 years (37). Brucellosis has been found in more than 170 countries in six major regions of the world (8). More than 500,000 new human infections are estimated to occur every year and more than 850 million pigs are infected with Brucella spp. (9, 10). At present, the prevalence of swine brucellosis varies widely worldwide, with the highest rates in America, North Africa and southern Europe $(11,12)$. In South America, the positive rate of swine brucellosis antibodies is $9 \%$, and some countries in the European Union have no swine brucellosis while other countries have a positive antibody rate of $22.7 \%$. In China, the positive rate of swine brucellosis antibodies in some areas is $10 \%(13-15)$. The prevalence of the disease varies among different regions, but the overall prevalence has been on the rise since the 1990s, which has had a considerable impact on the health of humans and animals and on the economy (16).

In addition to Brucella suis, there are 12 Brucella spp. currently (Brucella ovis, Brucella abortus, Brucella canis, et al.) and other strains without standing in nomenclature (17). Most of these species mainly infect specific hosts. Although it has been reported that pigs can be infected with different types of Brucella besides Brucella suis (18), B. suis is responsible for brucellosis in pigs. Brucella suis is composed of five biovars referred to as 1 through 5 (19, 20). Among them, Brucella suis biovars 1, 2 and 3 cause brucellosis in domestic swine, cattle, sheep and even human beings. Although Brucella suis is less harmful than Brucella melitensis and Brucella abortus, brucellosis in pigs caused by it often leads to chronic infection that is not easily detected (21). It may infect the surrounding livestock and other animals, increasing its epidemic scope and widening its range of infection (22). Most human infections derived from swine are caused by Brucella suis biovars 1 and 3 (23-27), which easily infect humans through direct exposure, particularly abattoir workers, farmers and veterinarians $(28,29)$. To date, there is no effective vaccine for Brucella (30). More importantly, the antimicrobial resistance of Brucella is emerging in brucellosis endemic regions of the world, such as China, Malaysia, Iran, Qatar and Egypt (31). Therefore, we should pay greater attention to its ongoing spread worldwide.

Pigs play a key role globally in providing animal protein in animal husbandry production. Pork is the most consumed land-animal meat, accounting for more than $36 \%$ of the world's meat intake, and has maintained a steady growth over the past few decades (32). Brucellosis was once considered to be one of the main diseases affecting the

Abbreviations: WHO, World Health Organization; LPS, Lipopolysaccharide; S-LPS, Smooth lipopolysaccharide; CNKI, Chinese National Knowledge Infrastructure; PRISMA, Preferred Reporting Items for Systematic Reviews and Meta-Analyses; OIE, Office International Des Epizooties. pig industry. In many countries, especially those in the developing world, pig production is usually housed in low biosecurity environments (32). However, as far as we know, systematic analyses of the overall prevalence of brucellosis in pigs worldwide, are scarce. Hence, we conducted a systematic review and meta-analysis of Brucella spp. infection worldwide to analyze the pooled prevalence of brucellosis in pigs and to assess potential risk factors associated with brucellosis prevalence.

\section{MATERIALS AND METHODS}

\section{Search Strategy}

Six databases were used to search the published research literature related to porcine brucellosis, including PubMed, ScienceDirect, SpringerLink, Web of Science, CNKI, Wanfang Data, and the VIP Chinese Journal Database. We retrieved all papers on worldwide Brucella spp. infection in swine that were published from January 1, 2000 to January 3, 2020 (the actual sampling dates in those publications were from 1980 to 2019).

In PubMed, the search terms and formulas used were "("Brucella suis" [MeSH] OR Brucella melitensis biovar suis) AND ("Swine"[MeSH] OR Suidae OR Pigs OR Warthogs OR Wart Hogs OR Hog, Wart OR Hogs, Wart OR Wart Hog OR Phacochoerus)." In ScienceDirect, we used the terms, "Brucella suis," "swine," "pig" and "prevalence." In SpringerLink, we used the terms "Brucella suis" and "pigs." In Web of Science, we used the keywords "Brucella suis," "Swine" and "prevalence" to search for the "TOPIC" (the article topic). We used the term "Brucella" (in Chinese) or "Brucella spp." (in Chinese) or "Brucellosis" (in Chinese) in the CNKI database. In Wanfang Data, we used the terms "Brucella" (in Chinese) and "pigs" (in Chinese), or "Brucella spp." (in Chinese) and "pigs" (in Chinese), or "Brucellosis" (in Chinese) and "pigs" (in Chinese). The types of articles found in Wanfang Data were limited to "papers in journals, degree theses and conferences." The search formulas used in the VIP Chinese Journal Database consisted of "Title" or "keywords" = "Brucella" (in Chinese) or "Brucellosis" (in Chinese) and "pigs" (in Chinese). The search strategies and search restrictions are reported in Supplementary Material 1. We used different keywords ("Brucella suis," "brucellosis," "swine," "pigs," "prevalence” and "epidemiological investigation") in each database for search verification; however, no additional qualified studies were found. Endnote (version X9.3.1) was used to catalog the articles retrieved.

Eligible studies were selected in accordance with the following criteria (inclusion criteria):

- The subjects of the research must be swine.

- The study's aim must be to investigate the prevalence of Brucella suis in swine.

- Data must include information on the number of examined pigs and the number of Brucella suis-positive pigs.

- The study must be published in Chinese or English. 
Articles with the following characteristics were excluded:

- Articles that did not match the titles and abstracts (see inclusion criteria).

- Repetition of articles or data.

- The hosts were not swine.

- The article was not research study.

- Unable to access the article's full text.

- Published before 2000.

- The article had one or more internal data conflicts.

- The number of samples was $<30$.

\section{Data Extraction and Quality Assessments of the Publications}

The four reviewers used standardized data collection forms to extract data that were consistent with the criteria to qualify for inclusion in the meta-analysis (33). Any differences between the reviewers or uncertainty about the quality of the research were resolved through the intervention of the lead author (QLG). The following information was reported: first author, the sampling year, the year of publication, income level, geographical region of the study, detection method, age, gender, collection season, feeding mode, pig classification, total number of pig samples and the number of samples that tested positive for Brucella.

The quality of the publications was graded using a scoring approach (34). We scored each study, and assigned a score of 5 when the information was described in greater detail (i.e., random sampling, detection method used, sampling method, sampling year and analyses of four or more factors). All the papers were assigned $0-5$ points based on the standards. The quality of the papers with 3,4 or 5 points was considered to be high, papers with a score of 2 points were considered to be average and those with 0 or 1 point were considered to be of low quality.

\section{Statistical Analysis}

Based on a large number of studies, all calculations, including the prevalence of Brucella spp. in swine were performed using $\mathrm{R}$ software (version 3.5.2). We chose the double-arcsine transformation (PFT) to perform the rate conversions (Table 1), based on these results and those of previous studies (35). The formula of PFT was as followed:

$$
\begin{aligned}
\mathrm{t} & =\arcsin \{\operatorname{sqrt}[\mathrm{r} /(\mathrm{n}+1)]\}+\arcsin \{\operatorname{sqrt}[(\mathrm{r}+1) /(\mathrm{n}+1)]\} \\
\operatorname{se}(\mathrm{t}) & =\operatorname{sqrt}[1 /(\mathrm{n}+0.5)] \\
\mathrm{p} & =[\sin (\mathrm{t} / 2)]^{2}
\end{aligned}
$$

Note: $\mathrm{t}$ : transformed prevalence; $r=$ positive number; $n=$ sample size; se $=$ standard error.

We used forest plots to visualize the results of the analyses and to evaluate the heterogeneity between the studies. Heterogeneity was calculated using Cochran's Q-test, the $\mathrm{I}^{2}$ statistic and the $\chi^{2}$ test $(P<0.05)$, and the cutoff value for the $\mathrm{I}^{2}$ statistic was $50 \%$. These two methods were used to examine the degree of statistical significance of the heterogeneity between the selected
TABLE 1 | Normal distribution test of the original rates and the different transformations of the original rates.

\begin{tabular}{lcc}
\hline Conversion form & W & P \\
\hline PRAW & 0.448 & $<2.2 \mathrm{e}-16$ \\
PLN & $\mathrm{NaN}$ & $\mathrm{NA}$ \\
PLOGIT & $\mathrm{NaN}$ & $\mathrm{NA}$ \\
PAS & 0.653 & $2.238 \mathrm{e}-15$ \\
PFT & 0.647 & $1.653 \mathrm{e}-15$ \\
\hline
\end{tabular}

PRAW, original rate; PLN, logarithmic conversion; PLOGIT, logit transformation; PAS, arcsine transformation; PFT, double-arcsine transformation; NaN, meaningless number; NA, missing data.

studies. We used a random effects model for the meta-analysis when heterogeneity was apparent in the selected articles (36). The funnel plot, trim and fill method and Egger's test were used to evaluate the studies for publication bias. Studies have shown that different subgroups may generate different funnel plots because of prevalence changes over time (37). Therefore, a funnel plot and forest plot were used for further evaluation of each subgroup. A sensitivity analysis was conducted to check whether any one study would have a significant impact on the estimates (38).

Heterogeneity between studies is an important indicator in meta-analyses; thus, an accurate assessment of heterogeneity is necessary to finding the key for preventing Brucella spp. infection in pigs worldwide. In order to examine the potential sources of heterogeneity, we analyzed the research data using subgroup analyses and univariate regression analysis to identify factors predictive of heterogeneity. The investigated factors consisted of geographical region (comparisons between Asia and other regions), the period of data collection (2006 to 2010 compared to 2000 or before, 2001 to 2005,2011 to 2015 and 2016 or later), income (comparisons of high- with low- and middle-incomes), detection methods (comparison of the RBPT \& TAT with other serological or molecular biology-based methods), season (comparisons of summer with spring, autumn and winter), gender (comparison of boars with sows), pigs' age classifications (comparisons of suckling pigs with finishing, growing and weaning pigs), feeding modes (comparison of extensive farms with intensive farms), pig classification (comparison of feral with domestic pigs) and quality of studies (comparisons of highquality studies with average-quality studies). This meta-analysis was performed in accordance with the PRISMA guidelines (Supplementary Material 2) (39-41). A correlation analysis was performed for each subgroup by detection method and country in order to track the source of heterogeneity. The heterogeneity of the covariates is represented by $\mathrm{R}^{2}$. Our meta-analysis does not include a review agreement and is not registered in the Cochrane database. The code in R for this meta-analysis was presented in Supplementary Material 3.

\section{RESULTS}

\section{Search Results and Quality of the Eligible Studies}

A total of 2,530 studies were retrieved from the seven databases. We conducted the meta-analysis with 119 studies based on our 


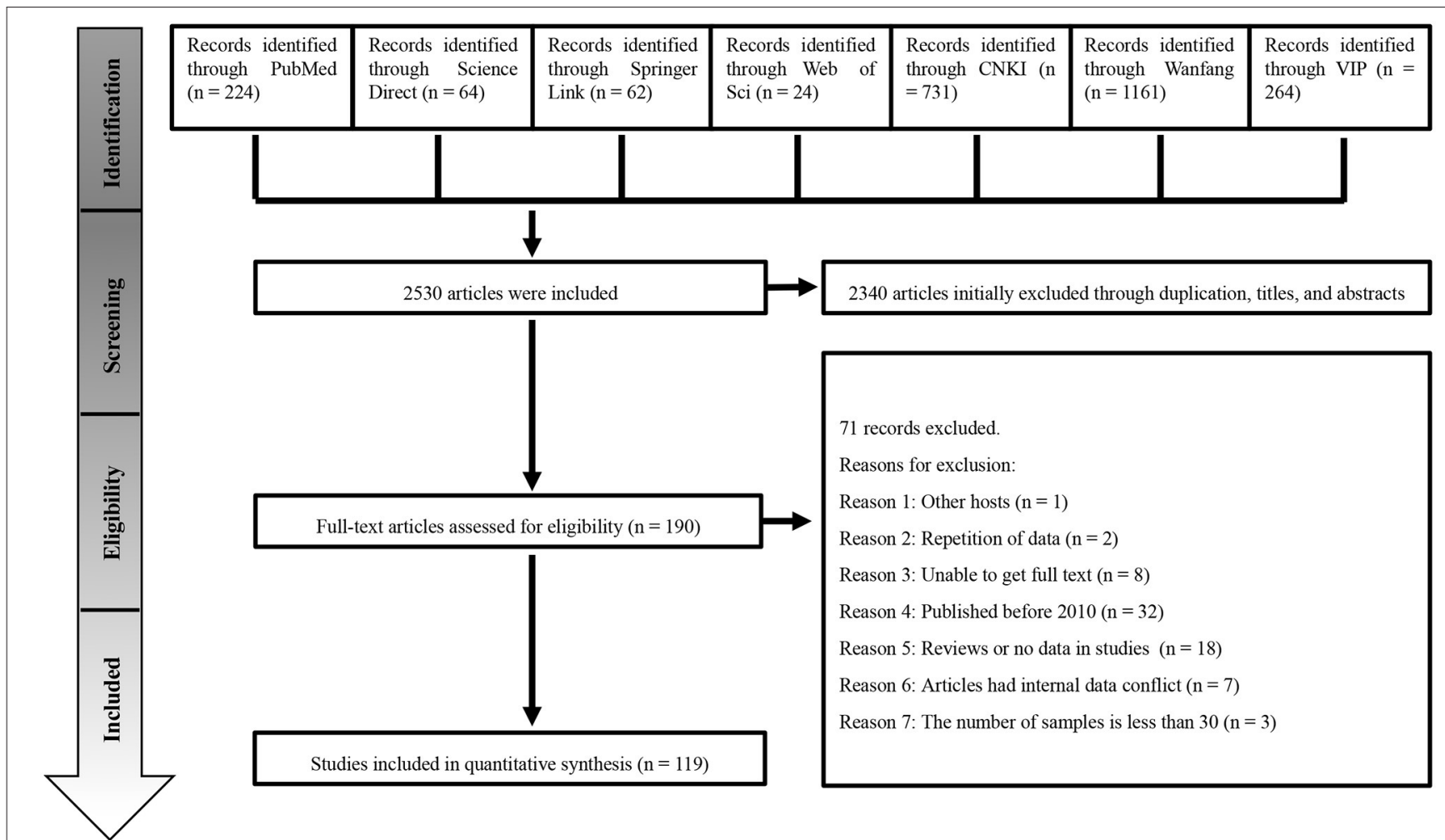

FIGURE 1 | Flow diagram of the search strategies and selection of studies.

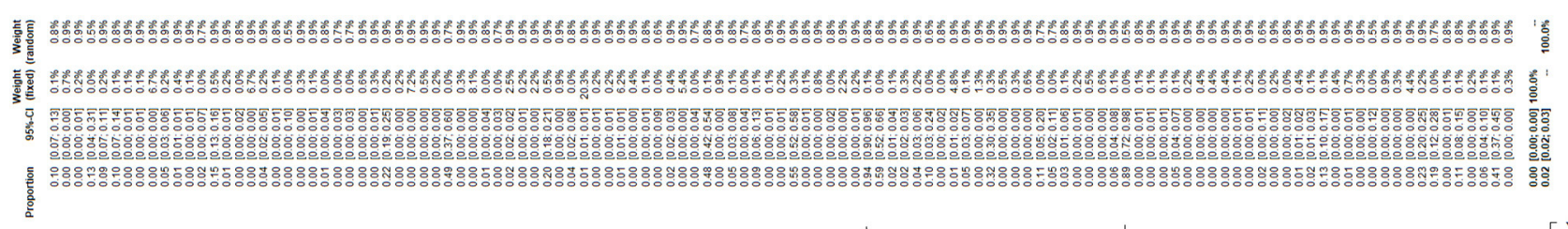

을
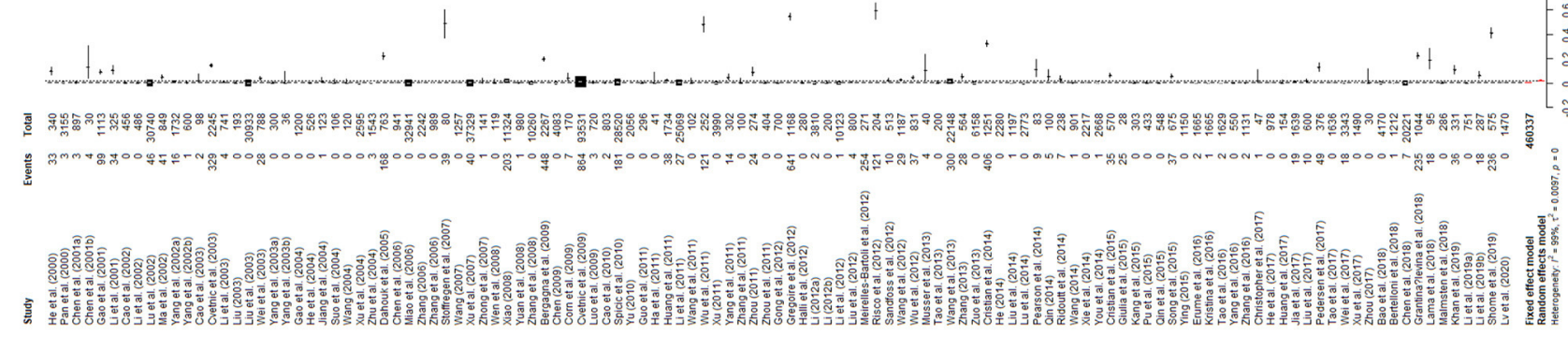

FIGURE 2 | Forest plot of the worldwide prevalence of Brucella spp. The length of the horizontal line represents the 95\% confidence interval, and the diamonds represent the summarized effect.

inclusion and exclusion criteria (Figure 1). The included articles consisted of 41 high-quality publications (4 or 5 points), 78 average-quality publications ( 2 or 3 points) and no low-quality publications (0 or 1 point; Supplementary Materials 4, 5).

\section{Results of Publication Bias}

The results of the forest plot showed a high degree of heterogeneity between studies $\left(\mathrm{I}^{2}=99.3 \%, P=0\right.$;
Figure 2). The funnel plot showed that the graph was asymmetric, indicating the possibility of publication bias or small study effects (Figure 3). Egger's test showed significant publication bias in the included studies $(P$ $<0.05$; Supplementary Materials 6, 7). The results of the trim and fill method showed that some studies were filled, indicating publication bias or small study effects (Supplementary Material 8). In addition, we evaluated 


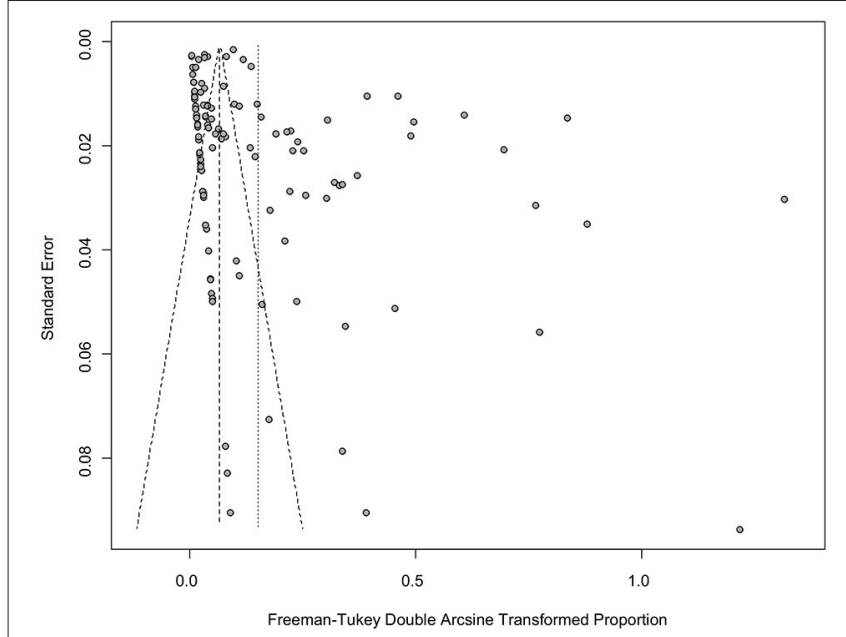

FIGURE 3 | Funnel plot with pseudo 95\% confidence intervals for the publication bias test.

publication bias in all subgroups using funnel plots (Supplementary Materials 9-18).

\section{Results of Sensitivity Analyses}

The results of the sensitivity analysis showed that when a study was omitted, the analysis of the remaining studies yielded the same results as the previous analysis. Therefore, the results of our systematic review and meta-analysis were relatively stable and reliable (Supplementary Material 19).

\section{Meta-Analysis of Brucella spp. in Swine worldwide}

Our meta-analysis included five global geographic regions, namely Africa, America, Asia, Europe and Oceania. The pooled prevalence of Brucella spp. in swine worldwide was 2.1\% (95\% CI: 1.6-2.6; Table 2). Among the regional subgroups, the highest prevalence occurred in Europe, which was 17.4\% (95\% CI: 11.124.9; Table 2). Among the countries, Brazil had the highest rate of $93.73 \%$ (95\% CI 90.5-96.3; Table 2), followed by Spain, with a rate of $59.3 \%$ (95\% CI: 52.5-66.0; Table 3).

We conducted a subgroup analysis of sampling years, income level, detection method, season, gender, age, feeding mode, pig classification and quality of studies to explore their influence on the prevalence of Brucella spp. in swine. Among them, regions, sampling years, income level, detection method, age of pigs, pig classification and quality of the study were identified as risk factors for Brucella spp. infection in pigs $(P<0.05$; Table 2). The combined prevalence of Brucella spp. in sampling years 2006 to 2010 was $2.7 \%$ (95\% CI: $1.1-4.8$; Table 2), which was higher than the other four periods. The estimate of prevalence in the high-income group was $15.7 \%$ (95\% CI: 8.0-25.3; Table 2), which was higher than that of the low- and middle-income groups. In the detection methods subgroup, the CFT showed a prevalence of $26.5 \%$ (95\% CI: $0.0-80.3$; Table 2). The point estimate of the prevalence of Brucella spp. in pigs during the summer was the highest at 11.1\% (95\% CI: 0.0-63.0; Table 2). Compared with the other ages of the pigs, the prevalence of Brucella spp. among the finishing pigs (4.9\%, 95\% CI: 0.9-11.0; Table 2) was higher than that of the growing pigs, suckling pigs and weaning pigs. The prevalence of Brucella spp. in feral pigs (15.0\%, 95\% CI: 8.423.2) was significantly higher than that of domestic pigs. The subgroup analysis by quality of study showed the prevalence of Brucella spp. in swine was higher in the studies of high quality (5.0\%, 95\% CI: 3.5-6.7; Table 2). The heterogeneity of each subgroup was explained by detection method (the covariate), which ranged from $0-79.25 \%\left(\mathrm{R}^{2}\right.$-method), and countries (the covariate), which was $60.97-97.04 \%\left(\mathrm{R}^{2}\right.$-country).

\section{DISCUSSION}

Brucellosis is a zoonotic infectious disease caused by Brucella. It is the main cause of infertility, low litter size and miscarriage among sows (42) and an occupational hazard for farmers, slaughterhouse workers and veterinarians $(43,44)$. The OIE, WHO and Food and Agriculture Organization of the United Nations have classified brucellosis as one of the most important neglected occupational hazards in the world $(45,46)$. It has had a significant economic impact on the livestock industry and other industries (47). The prevalence of brucellosis plays an important role in the development of the world's pig herds (48). Therefore, we conducted the first meta-analysis to examine the prevalence of brucellosis in pig herds around the world and found that it was unevenly distributed among pigs.

Brucellosis in swine has been widely distributed worldwide for a long time, but in some high-income countries, including Canada, Australia, New Zealand and other countries, the eradication of brucellosis in animal husbandry has been successfully achieved $(49,50)$. Brucellosis has not been reported in domestic pigs in Belgium since 1969 (51). The United States has implemented reforms in pig management since 1950 to eliminate brucellosis in livestock, and efforts have been made to solve the problem of brucellosis infection in wild animals with almost complete eradication of it in livestock populations (52). However, in our study, the highest prevalence rates were found in America and Europe, and the prevalence rates in the high-income countries were higher than those in middleand low-income countries. We attribute these results to several factors. First, most of the samples tested in these countries were feral or domestic pigs in contact with wild boars. This was confirmed in the subgroup analysis of pig classification, which showed the prevalence of feral pigs was significantly higher than that of domestic pigs. In recent decades, the population of wild boars has increased rapidly in the United States, which is caused mainly by natural population dynamics, and brucellosis has been reported in wild boars in 14 states (53). In Belgium, the increase in the wild boar population and the prevalence of brucellosis has led to an increased risk of infection in outdoor pig farms (54). Therefore, overabundance of wildlife is considered to be an important factor in the transmission of brucellosis between wildlife and livestock (55). After the first isolation of the Brucella suis biovar 2 strain from boars killed by hunters 
TABLE 2 | Pooled worldwide prevalence of Brucella spp. by region.

No. studies No. tested No positive

\section{Heterogeneity}

\section{Regions*}

Africa

America

Asia

Europe

Oceania

Sampling years

2000 or

before

2001 to 2005

2006 to 2010

2011 to 2015

2016 or later

Income level*

Low

Middle

High

Detection method*

CFT

ELISA

PCR

RBPT

RBPT \& TAT

TAT

Others

Season*

Spring
Summer

Autumn

Winter

Gender

Boars

Sows

Age of pigs

Finishing pigs

Growing pigs
3,661

2,570

320,164

133,621

321

$41 \quad 81,246$

42

35

31

12

2

95

22

6

6
11

11
3

3
55

55
23

23
17

26

11
5

5
6

6

140,647

\section{5,798}

32,998

8,965

3,330

445,062

11,945

2,174

35,170

623

173,023

105,223

20,121

112,102

\section{3,716}

1,163

1,452

3,444

12,737

51,698

5,778

31,454 $\%\left(95 \% \mathrm{Cl}^{\star}\right)$

$P$ - $\quad I^{2}(\%) \quad P$-value $\quad$ Coefficient $(95 \% \mathrm{Cl})$

\begin{tabular}{|c|c|c|c|}
\hline $1.7 \%(0.0-6.7)$ & 111.64 & $<0.01$ & $98.2 \%$ \\
\hline $16.5 \%(1.3-42.8)$ & $1,580.03$ & 0.00 & $99.5 \%$ \\
\hline $0.5 \%(0.3-0.7)$ & $4,029.49$ & 0.00 & $97.8 \%$ \\
\hline $17.4 \%(11.1-24.9)$ & $7,805.04$ & 0.00 & $99.8 \%$ \\
\hline $6.0 \%(0.5-16.0)$ & 6.73 & $<0.01$ & $85.1 \%$ \\
\hline $0.7 \%(0.3-1.4)$ & $2,252.12$ & 0.00 & $98.2 \%$ \\
\hline $0.7 \%(0.4-1.1)$ & $2,182.60$ & $<0.01$ & $98.1 \%$ \\
\hline $2.7 \%(1.1-4.8)$ & $4,111.44$ & 0.00 & $99.2 \%$ \\
\hline $0.6 \%(0.1-1.3)$ & $1,154.60$ & $<0.01$ & $97.4 \%$ \\
\hline $0.7 \%(0.0-2.0)$ & 289.89 & $<0.01$ & $96.2 \%$ \\
\hline $0.1 \%(0.0-0.2)$ & 0.27 & - & - \\
\hline $0.8 \%(0.5-1.1)$ & $7,877.36$ & 0.00 & $98.8 \%$ \\
\hline $15.7 \%(8.0-25.3)$ & $3,438.12$ & 0.00 & $99.4 \%$ \\
\hline $26.5 \%(0.0-80.3)$ & $1,682.83$ & $<0.01$ & $99.7 \%$ \\
\hline $9.4 \%(2.1-21.0)$ & $3,647.44$ & 0.00 & $99.7 \%$ \\
\hline $16.3 \%(5.0-32.2)$ & 41.30 & $<0.01$ & $95.2 \%$ \\
\hline $1.4 \%(0.8-2.1)$ & $5,727.35$ & 0.00 & $99.1 \%$ \\
\hline $0.2 \%(0.1-0.4)$ & 479.88 & $<0.01$ & $95.4 \%$ \\
\hline $0.5 \%(0.0-1.4)$ & 312.29 & $<0.01$ & $94.9 \%$ \\
\hline $11.0 \%(6.1-17.2)$ & 6,830.99 & 0.00 & $99.6 \%$ \\
\hline $2.6 \%(0.0-3.8)$ & 226.43 & $<0.01$ & $95.6 \%$ \\
\hline $11.1 \%(0.0-63.0)$ & $1,300.31$ & $<0.01$ & $99.7 \%$ \\
\hline $0.7 \%(0.0-3.8)$ & 46.65 & $<0.01$ & $89.3 \%$ \\
\hline $1.2 \%(0.1-3.3)$ & 63.17 & $<0.01$ & $92.1 \%$ \\
\hline $7.9 \%(2.4-15.9)$ & $3,594.80$ & 0.00 & $99.4 \%$ \\
\hline $5.1 \%(2.8-8.1)$ & 3,208.62 & 0.00 & $99.1 \%$ \\
\hline $4.9 \%(0.9-11.0)$ & 684.93 & $<0.01$ & 98.1 \\
\hline $2.1 \%(0.2-5.2)$ & 1271.98 & $<0.01$ & 9 \\
\hline
\end{tabular}

Joint analysis*

$\mathbf{R}^{2}$-method $\quad \mathbf{R}^{2}$-country

$<0.001$

$0.00 \%$

$68.49 \%$

3,575

16

$$
922
$$

1,182

1,095

374

$$
82
$$

$$
3
$$

3,093

2,345

295

1,216

101

1,846

162

275

3,169

$$
69
$$

$$
\begin{array}{r}
69 \\
258
\end{array}
$$$$
12
$$

$$
21
$$

$$
1282
$$

1288

321

411

$<0$

$0.297(0.256$ to 0.338$)$

$19.42 \%$

$70.65 \%$

$\begin{array}{lll}<0.001 & -0.152(-0.206 \text { to }-0.103) & 0.00 \%\end{array} 66.46 \%$

$0.195(-0.021$ to 0.411$) \quad 36.17 \% \quad 97.04 \%$

$0.056(-0.053$ to 0.165$)$

$82.75 \%$ 
TABLE 2 | Continued

\begin{tabular}{|c|c|c|c|c|c|c|c|c|c|c|c|}
\hline & \multirow[t]{2}{*}{ No. studies } & \multirow[t]{2}{*}{ No. tested } & \multirow[t]{2}{*}{ No. positive } & \multirow[t]{2}{*}{$\%\left(95 \% \mathrm{Cl}^{\star}\right)$} & \multicolumn{3}{|c|}{ Heterogeneity } & \multicolumn{2}{|c|}{ Univariate meta-regression } & \multicolumn{2}{|c|}{ Joint analysis* } \\
\hline & & & & & $x^{2}$ & $\begin{array}{c}P- \\
\text { value }\end{array}$ & $\mathrm{I}^{2}(\%)$ & $P$-value & Coefficient (95\% Cl) & $\mathrm{R}^{2}$-method & $\mathbf{R}^{2}$-country \\
\hline Suckling pigs & 9 & 1,917 & 7 & $0.0 \%(0.0-0.5)$ & 15.01 & 0.05 & $46.7 \%$ & 0.045 & $-0.120(-0.237$ to -0.003$)$ & $79.25 \%$ & $87.44 \%$ \\
\hline Weaning pigs & 5 & 1,015 & 28 & $1.0 \%(0.0-14.2)$ & 98.67 & $<0.01$ & $95.9 \%$ & & & & \\
\hline \multicolumn{12}{|l|}{ Feeding mode } \\
\hline Extensive & 12 & 34,083 & 555 & $2.5 \%(0.4-5.9)$ & $1,823.79$ & 0.00 & $99.2 \%$ & 0.065 & $0.076(-0.005$ to 0.156$)$ & $6.50 \%$ & $68.57 \%$ \\
\hline Intensive & 35 & 55,196 & 468 & $0.5 \%(0.1-1.2)$ & $1,790.10$ & 0.00 & $98.9 \%$ & & & & \\
\hline \multicolumn{12}{|l|}{ Pig classification } \\
\hline $\begin{array}{l}\text { Domestic } \\
\text { pigs }\end{array}$ & 21 & 131,196 & 1,504 & $1.1 \%(0.2-2.5)$ & $2,397.46$ & 0.00 & $99.5 \%$ & & & & \\
\hline Feral pigs & 21 & 9,186 & 2,085 & $15.0 \%(8.4-23.2)$ & $1,838.57$ & 0.00 & $99.0 \%$ & $<0.001$ & 0.277 (0.197 to 0.357$)$ & $0.00 \%$ & $74.40 \%$ \\
\hline \multicolumn{12}{|l|}{ Quality level } \\
\hline Middle & 78 & 203,157 & 1,201 & $1.0 \%(0.6-1.3)$ & $4,344.03$ & 0.00 & $98.2 \%$ & & & & \\
\hline High & 41 & 257,180 & 4,240 & $5.0 \%(3.5-6.7)$ & $11,773.05$ & 0.00 & $99.7 \%$ & $<0.001$ & 0.117 (0.078 to 0.157$)$ & $9.21 \%$ & $60.97 \%$ \\
\hline Total & 119 & 460,337 & 5,441 & $2.1 \%(1.6-2.6)$ & $16,698.88$ & 0.000 & $99.3 \%$ & & & & \\
\hline
\end{tabular}

$\mathrm{Cl}^{*}$, Confidence interval; Joint analysis", Joint analysis with prevalence of detection methods and provinces in China; $R^{2}$, Proportion of between-study variance explained.

Region*: Africa: India, Uganda; America: Brazil. USA; Asia: China. India; Europe: Belgium, Croatia, Finland, Germany, Italy, Latvia, Sweden; Oceania: Australia.

Method": CFT: Complement fixation test; ELISA: Enzyme linked immunosorbent assay; PCR: Polymerase chain reaction; RBPT: Rose Bengal plate test; RBPT\&TAT: Rose Bengal plate test and Tube agglutination test; TAT: Tube agglutination test.

Season*: Spring: Mar. to May.; Summer: Jun. to Aug.; Autumn: Sep. to Nov.; Winter: Dec. to Feb.

Income level: High: Developed Country; Middle: Developing Country; Low: Least Developed Country. 
TABLE 3 | Estimated pooled seroprevalence of Brucella spp. by country and region worldwide.

\begin{tabular}{|c|c|c|c|c|c|c|}
\hline Countries & No. studies & Region & No. tested & No. positive & $\%$ Prevalence & $\%(95 \%$ Cl) \\
\hline Australia & 2 & Oceania & 321 & 16 & $6.0 \%$ & $0.5-16.0$ \\
\hline Belgium & 1 & Europe & 1,168 & 641 & $54.9 \%$ & $52.0-57.7$ \\
\hline Brazil & 1 & America & 271 & 254 & $93.7 \%$ & $90.5-96.3$ \\
\hline China & 89 & Asia & 319,589 & 1,193 & $0.3 \%$ & $0.2-0.5$ \\
\hline Croatia & 3 & Europe & 124,296 & 1,374 & $3.5 \%$ & $1.3-6.8$ \\
\hline Germany & 1 & Europe & 763 & 168 & $22.0 \%$ & $19.2-25.0$ \\
\hline India & 1 & Asia & 575 & 236 & $41.0 \%$ & $37.1-45.1$ \\
\hline Italy & 5 & Europe & 5,328 & 915 & $22.6 \%$ & $6.7-44.3$ \\
\hline Spain & 1 & Europe & 204 & 121 & $59.3 \%$ & $52.5-66.0$ \\
\hline Latvia & 1 & Europe & 1,044 & 235 & $22.5 \%$ & $20.0-25.1$ \\
\hline USA & 8 & America & 2,299 & 128 & $8.7 \%$ & $2.0-19.2$ \\
\hline Total & 119 & & 460,337 & 5,441 & $2.1 \%$ & $1.6-2.6$ \\
\hline
\end{tabular}

in 1994 (56), Brucella suis biovar 2 has been isolated from wild boars in many countries (57-61). Studies have reported that brucellosis among wild boars is widely distributed all over the world (51, 56, 57, 62-65). Second, different modes of feeding in different countries have led to different prevalence rate. Most developed countries mainly focus on intensive farming, while countries with lower incomes focus mainly focus on extensive farming. The prevalence of disease in countries with intensive farming is higher than that in countries with extensive farming, which has been confirmed in several studies (66-68). The increase in herd size results in higher stocking density and worse farm sanitation, thereby promoting the spread of Brucella among animals after abortion and parturition $(69,70)$. We recommend long-term monitoring of wildlife to implement preventive measures before an outbreak of brucellosis. Intensive farms need to control breeding density, pay attention to animal welfare, improve the prevention and control of epidemics and optimize the breeding environment to avoid the large-scale spread of disease. It is worth noting that among the studies we included, only a few on swine brucellosis were conducted in low-income countries. This may indicate that swine brucellosis has been overlooked in these countries and regions. Therefore, although our results show that the prevalence of brucellosis in low-income countries is lower than that of other countries, this finding may be due to these countries' neglect of surveillance and detection of brucellosis. Likewise, the farms in high-income countries are more capable of strengthening their detection of brucellosis, thus, showing a relatively high prevalence. We infer that the actual global infection rate of swine brucellosis may be higher. Although the disease has been controlled or eliminated in some developed countries (such as Canada, New Zealand, Australia and the majority of northern European countries) (71), brucellosis remains an intractable public health problem in poor and underdeveloped countries, especially in the Middle East (72). While strengthening the surveillance and prevention of swine brucellosis in high-incidence areas, we should also continue to strengthen the surveillance in lower incidence areas to prevent widespread infection of swine brucellosis.

The 2006-2010 prevalence of brucellosis was higher than that of the other sampling years. First, as reported in the included studies, an outbreak of swine brucellosis in Jaboticabal, Brazil in 2006 increased the prevalence to $93.7 \%$ (73). At the same time, brucellosis was found in Italy after collecting samples from pig farms with breeding problems for serological analysis. Furthermore, detection of suspected cases (non-random sampling) may overestimate the local prevalence of brucellosis $(74,75)$. Second, during this period, many countries began to analyze the situation of Brucella infection in feral pigs. Among the included articles, there were 21 studies on the prevalence of wild boars, and the prevalence of wild boars between 2006 and 2010 was $22.3 \%(881 / 3956)$. There were also studies on isolated Brucella suis biovar 2 from wild boars because of the substantial increase in the number of feral pigs (58-60, 76). Moreover, several Brucella outbreaks occurred in Germany due to infection of domestic pigs by feral pigs (77). After 2010, the prevalence of brucellosis gradually declined because the OIE proposed controls for Brucella farms, giving priority to the development of food safety standards for future animal production (78). Although the prevalence of brucellosis has shown a downward trend, its control should be continued.

The 119 selected studies that were analyzed included five main methods of brucellosis detection: CFT, ELISA, PCR, RBPT and TAT $(P<0.001$; Table 2$)$. We used detection method as a covariate to perform joint analysis with other risk factors, and the range of heterogeneity explained by the detection method was 0 $100.00 \%$, implying that different detection methods had a greater effect on some subgroups.

The analyses of the age and sex of pigs showed that the prevalence of finishing pigs was higher than that of the pigs in the other age groups, and the prevalence of sows was lower than 
that of the boars. This finding is mainly due to the adult males' contacts with these matrilineal groups during the mating season, while females live in matrilineal groups (60). The prevalence observed in the finishing pigs was higher than that in the other age groups, which was due to the higher involvement of the finishing pigs (79). Although the prevalence of boars was higher than that of sows, no significant differences were found between them, indicating that the relationship between the two animals warrants further examination. We recommend controlling the breeding density; planning a reasonable breeding process may play a positive role in the reduction of the spread of brucellosis in pigs.

The prevalence of brucellosis in the summer was higher than that in the other seasons, but the difference was not significant (Table 2). As far as we know, no research has shown a strong correlation between breeding season and prevalence of swine brucellosis. Studies have shown that the dryness of summer may lead to lack of food and water, increasing the trajectory coverage of animals (80). The correlation analysis showed that countries explained $97.04 \%$ of heterogeneity in the seasons subgroup. These results can be interpreted in the context of the world's vast territory with different countries having different characteristics during summer. Meta-analyses showed that the incidence rates of Brucella in cattle and deer were higher in hotter and more humid areas $(81,82)$. Therefore, we speculate that a similar phenomenon occurs in pigs with brucellosis. Thus, efforts to prevent epidemics should be increased in hot and humid areas to create a healthy environment for livestock and reduce the occurrence of disease.

Our study included 41 high-quality articles and 78 averagequality articles. We found that random sampling and detailed descriptions of sampling methods were not included in some of the articles by examining those of average quality. These findings may reflect sampling bias. We recommend that researchers provide detailed descriptions of their sampling and data collection methods to improve the reliability of the data.

This meta-analysis had the advantages of a long-time span, wide coverage and clear methods of analyses, yet it has some limitations. First, the language of the selected articles was limited to English or Chinese, and therefore, qualified articles in other languages might have been overlooked. Second, the articles were obtained from seven databases, which might have excluded qualified articles from other databases. Third, the inadequate information provided by the included studies (e.g., brucellosis classification and geographical factors) might have led to publication bias or other biases in the subgroups (Supplementary Figures 3-12). Fourth, some risk factors were examined in a small number of studies and samples, which might have resulted in small study effects leading to unstable results. We recommend that researchers conduct large-scale studies because the results of small-scale studies are often not representative of the population. Fifth, the research we have included covers only 14 countries, and some of those countries (e.g., Brazil) had few relevant reports. For these countries, we only presented data to reflect global trends, and the results presented are for reference only. The lack of articles from some countries might have led to inaccurate estimates of the prevalence of swine brucellosis in those countries. Prevalence surveys of Brucella spp. in more countries are recommended to clarify the true prevalence of swine brucellosis worldwide. Sixth, this study was not registered; however, it was carried out strictly in accordance with the PRISMA guidelines.

In conclusion, the Brucella infection rate in pig herds is distributed widely throughout the world. In addition, Brucella is common among wild boars in developed countries. Therefore, we suggest carrying out long-term detection of Brucella in wild animals and implementing reasonable isolation measures between livestock and wild animals to reduce the chance of contact between them. In addition, countries that do not pay much attention to swine brucellosis should disseminate information about Brucella infection, and epidemiological investigations should be conducted as soon as possible to establish better control of the disease. The high prevalence of swine brucellosis will cause serious economic losses to herdsmen, and increase the risk of infection. Therefore, attention to animal welfare on intensive pig farms is crucial, and control of the breeding density may play an important role in reducing the spread of brucellosis in pigs. This study can provide a theoretical basis for researchers to explore control schemes for brucellosis.

\section{DATA AVAILABILITY STATEMENT}

The original contributions presented in the study are included in the article/Supplementary Material, further inquiries can be directed to the corresponding author/s.

\section{AUTHOR CONTRIBUTIONS}

YZ and RD: idea contributions and funding. BZ, G-YG, Z-YC, and YY: data extraction. Y-HS: database establishment. QW and J-ML: data analysis. Q-LG: writing - original draft. Y-HS, KS, and $\mathrm{XL}$ : writing - review and editing. All authors contributed to the article and approved the submitted version.

\section{FUNDING}

This study was supported by the National Key R \& D Program of China (2018YFD0500900) and the Scientific Research Planning Project of the Jilin Provincial Department of Education (JJKH20200364KJ).

\section{ACKNOWLEDGMENTS}

We thank the scientists and personnel of the College of Animal Science and Technology, Jilin Agricultural University and the College of Chinese Medicine Materials, Jilin Agricultural University, for their collaboration.

\section{SUPPLEMENTARY MATERIAL}

The Supplementary Material for this article can be found online at: https://www.frontiersin.org/articles/10.3389/fvets. 2021.630960/full\#supplementary-material 


\section{REFERENCES}

1. Franco-Paredes C, Chastain D, Taylor P, Stocking S, Sellers B. Boar hunting and brucellosis caused by Brucella suis. Travel Med Infect Dis. (2017) 16:18-22. doi: 10.1016/j.tmaid.2017.03.006

2. Gusi AM, Bertu WJ, Jesús de Miguel M, Dieste-Pérez L, Smits HL, Ocholi RA, et al. Comparative performance of lateral flow immunochromatography, iELISA and Rose Bengal tests for the diagnosis of cattle, sheep, goat and swine brucellosis. PLoS Negl Trop Dis. (2019) 13:e0007509. doi: 10.1371/journal.pntd.0007509

3. Hammerl JA, Ulrich RG, Imholt C, Scholz HC, Jacob J, Kratzmann N, et al. Molecular survey on brucellosis in rodents and shrews - natural reservoirs of novel Brucella species in Germany? Transbound Emerg Dis. (2017) 64:663-71. doi: $10.1111 /$ tbed.12425

4. Zhang N, Huang D, Wu W, Liu J, Liang F, Zhou B, et al. Animal brucellosis control or eradication programs worldwide: a systematic review of experiences and lessons learned. Prev Vet Med. (2018) 160:105-15. doi: 10.1016/j.prevetmed.2018.10.002

5. Buhmann G, Paul F, Herbst W, Melzer F, Wolf G, Hartmann K, et al. Canine brucellosis: Insights into the epidemiologic situation in Europe. Front Vet Sci. (2019) 6:151. doi: 10.3389/fvets.2019.00151

6. Kauffman LK, Petersen CA. Canine brucellosis: old foe and reemerging scourge. Vet Clin North Am Small Anim Pract. (2019) 49:763-79. doi: 10.1016/j.cvsm.2019.02.013

7. Kawakami N, Wakai Y, Saito K, Imaoka K. Chronic brucellosis in Japan. Intern Med. (2019) 58:3179-83. doi: 10.2169/internalmedicine.2961-19

8. Deng Y, Liu X, Duan K, Peng Q. Research progress on brucellosis. Curr Med Chem. (2018) 26:5598-608. doi: 10.2174/0929867325666180510125009

9. de Figueiredo P, Ficht TA, Rice-Ficht A, Rossetti CA, Adams LG. Pathogenesis and immunobiology of brucellosis: review of Brucella-host interactions. Am J Pathol. (2015) 185:1505-17. doi: 10.1016/j.ajpath.2015.03.003

10. Liu F. Construction and evaluation of the immunogenicity of Brucella S2BCSP31 deletion strain for vaccine (In Chinese) (Doctoral Dissertation), Jilin Agricultural University, Changchun (2018).

11. Pappas G, Akritidis N, Bosilkovski M, Tsianos E. Brucellosis. New Engl J Med. (2005) 352:2325. doi: 10.1056/NEJMra050570

12. Buzgan T, Karahocagil MK, Irmak H, Baran AI, Karsen H, Evirgen O.Clinical manifestations and complications in 1028 cases of brucellosis: a retrospective evaluation and review of the literature. Int J Infect Dis. (2010) 14:e469-78. doi: 10.1016/j.ijid.2009.06.031

13. Sandfoss MR, DePerno CS, Betsill CW, Palamar MB, Erickson G, KennedyStoskopf S. A serosurvey for Brucella suis, classical swine fever virus, porcine circovirus type 2, and pseudorabies virus in feral swine (Sus scrofa) of eastern North Carolina. J Wildl Dis. (2012) 48:462-6. doi: 10.7589/0090-3558-48.2.462

14. Grantina-Ievina L, Avsejenko J, Cvetkova S, Krastina D, Streikisa M, Steingolde Z, et al. Seroprevalence of Brucella suis in eastern Latvian wild boars (Sus scrofa). Acta Vet Scand. (2018) 60:19. doi: 10.1186/s13028-018-0373-9

15. Li L, Yan JH, Yan H, LI JP, MI JT, Di LXT. Research of prevention technology and prevalent in livestock brucellosis in Xinjiang (In Chinese). Grass-Feeding Livestock. (2012) 3:20-2. doi: 10.16863/j.cnki.1003-6377.2012.03.005

16. Avila-Granados LM, Garcia-Gonzalez DG, Zambrano-Varon JL, ArenasGamboa AM. Brucellosis in Colombia: Current status and challenges in the control of an endemic disease. Front Vet Sci. (2019) 6:321. doi: 10.3389/fvets.2019.00321

17. Acka PG, Zakowska D, Naylor K, Niemcewicz M, Bielawska-drozd A. Brucella -Virulence Factors, Pathogenesis and Treatment. Pol J Microbiol. (2018) 67:151-61. doi: 10.21307/pjm-2018-029

18. Stoffregen WC, Olsen SC, Wheeler CJ, Bricker BJ, Palmer MV, Allen E, et al. Diagnostic characterization of a feral swine herd enzootically infected with Brucella. J Vet Diagn Invest. (2007) 19:227-37. doi: 10.1177/104063870701900301

19. Sankarasubramanian J, Vishnu US, Gunasekaran P, Rajendhran J. A genome-wide SNP-based phylogenetic analysis distinguishes different biovars of Brucella suis. Infect Genet Evol. (2016) 41:213-7. doi: 10.1016/j.meegid.2016.04.012

20. More S, Bøtner A, Butterworth A, Calistri P, Depner K, Edwards S, et al. Assessment of listing and categorisation of animal diseases within the framework of the Animal Health Law (Regulation (EU) No 2016/429): paratuberculosis. Efsa J. (2017) 15:e04960. doi: 10.2903/j.efsa.2017.4960
21. Olsen SC, Tatum FM. Swine brucellosis: current perspectives. Vet Med. (2016) 8:1-12. doi: 10.2147/VMRR.S91360

22. Szulowski K, Iwaniak W, Weiner M, Złotnicka J. Brucella suis biovar 2 isolations from cattle in Poland. Ann Agric Environ Med. (2013) 20:672-5.

23. Menshawy AM, Perez-Sancho M, Garcia-Seco T, Hosein HI, García N, Martinez I, et al. Assessment of genetic diversity of zoonotic Brucella spp. recovered from livestock in Egypt using multiple locus VNTR analysis Biomed Res Int. (2014) 2014:353876. doi: 10.1155/2014/353876

24. Tian GZ, Cui BY, Piao DR, Zhao HY, Li LY, Liu X, et al. Multi-locus variablenumber tandem repeat analysis of Chinese Brucella strains isolated from 1953 to 2013. Infect Dis Poverty. (2017) 6:89. doi: 10.1186/s40249-017-0296-0

25. De Massis F, Zilli K, Di Donato G, Nuvoloni R, Pelini S, Sacchini L, et al. Distribution of Brucella field strains isolated from livestock, wildlife populations, and humans in Italy from 2007 to 2015. PLoS One. (2019) 14:e0213689. doi: 10.1371/journal.pone.0213689

26. Liu ZG, Wang M, Zhao HY, Piao DR, Jiang H, Li ZJ. Investigation of the molecular characteristics of Brucella isolates from Guangxi province, China. BMC Microbiol. (2019) 19:292. doi: 10.1186/s12866-019-1665-6

27. Wang XM, Huang MH, Cui BY. Analysis on brucellosis epidemiological characteristics in Hainan province. Chin J Epidemiol. (2019) 40:350-3.

28. Galińska EM, Zagórski J. Brucellosis in humans-etiology, diagnostics, clinical forms. Ann Agric Environ Med. (2013) 20:233-8.

29. Zheng R, Xie S, Lu X, Sun L, Zhou Y, Zhang Y, et al. A systematic review and meta-analysis of epidemiology and clinical manifestations of human brucellosis in China. Biomed Res Int. (2018) 2018:5712920. doi: $10.1155 / 2018 / 5712920$

30. Zriba S, Garcia-Gonzalez DG, Khalaf OH, Wheeler L, Chaki SP, Rice-Ficht A, et al. Vaccine safety studies of Brucella abortus S19 and S19 $\Delta$ vjbR in pregnant swine. Vaccine X. (2019) 3:100041. doi: 10.1016/j.jvacx.2019.100041

31. Solís, J., and Solera, J. (2015). Treatment of Human Brucellosis - Review of Evidence from Clinical Trials.

32. Beltran-Alcrudo D, Falco JR, Raizman E, Dietze K. Transboundary spread of pig diseases: the role of international trade and travel. BMC Vet Res. (2019) 15:64. doi: 10.1186/s12917-019-1800-5

33. Wang W, Gong QL, Zeng A, Li MH, Zhao Q, Ni HB. Prevalence of Cryptosporidium in pigs in China: A systematic review and meta-analysis. Transbound Emerg Dis. (2020). doi: 10.1111/tbed.13806. [Epub ahead of print].

34. Ran X, Cheng J, Wang $M$, Chen $X$, Wang $H$, Ge $Y$, et al. Brucellosis seroprevalence in dairy cattle in China during 2008-2018: A systematic review and meta-analysis. Acta Trop. (2019) 189:117-23. doi: 10.1016/j.actatropica.2018.10.002

35. Barendregt JJ, Doi S, Lee YY, Pacella R, Vos T. Meta-analysis of prevalence. J Epidemiol Commun H. (2013) 67:974-8. doi: 10.1136/jech-2013-203104

36. Assefa A, Bihon A. Bovine cysticercosis in Ethiopia: A systematic review and meta-analysis of prevalence from abattoir-based surveys. Prev Vet Med. (2019) 169:104707. doi: 10.1016/j.prevetmed.2019.104707

37. Ni HB, Gong QL, Zhao Q, Li XY, Zhang XX. Prevalence of Haemophilus parasuis "Glaesserella parasuis" in pigs in China: A systematic review and meta-analysis. Prev Vet Med. (2020) 182:105083. doi: 10.1016/j.prevetmed.2020.105083

38. Gong QL, Li D, Diao NC, Liu Y, Li BY, Tian T, et al. Mink Aleutian disease seroprevalence in China during 1981-2017: A systematic review and meta-analysis. Microb Pathog. (2020) 139:103908. doi: 10.1016/j.micpath.2019.103908

39. Moher D, Liberati A, Tetzlaff J, Altman DG. Preferred reporting items for systematic reviews and meta-analyses: the PRISMA statement. Int J Surg. (2010) 8:336-41. doi: 10.1016/j.ijsu.2010.02.007

40. Moher D, Shamseer L, Clarke M, Ghersi D, Liberati A, Petticrew M, et al. Preferred reporting items for systematic review and meta-analysis protocols (PRISMA-P) 2015 statement. Syst Rev. (2015) 4:1. doi: 10.1186/2046-4053-4-1

41. Shamseer L, Moher D, Clarke M, Ghersi D, Liberati A, Petticrew M, et al. Preferred reporting items for systematic review and meta-analysis protocols (PRISMA-P) 2015: elaboration and explanation. BMJ. (2015) 350:g7647. doi: 10.1136/bmj.g7647

42. Megid J. Clinical manifestations of brucellosis in domestic animals and humans. Open Vet Sci J. (2010) 4:119-26. doi: 10.2174/18743188010040 10119

43. Radostits OM, Blood DC, Done SH. Veterinary medicine: a textbook of the diseases of cattle, sheep, pigs, goats and horses. J Equine Vet Sci. (1994) 20:625. 
44. Swai ES, Schoonman L. A survey of zoonotic diseases in trade cattle slaughtered at Tanga city abattoir: a cause of public health concern. Asian Pac J Trop Biomed. (2012) 2:55-60. doi: 10.1016/S2221-1691(11) 60190-1

45. Musallam II, Abo-Shehada MN, Hegazy YM, Holt HR, Guitian FJ. Systematic review of brucellosis in the middle east: disease frequency in ruminants and humans and risk factors for human infection. Epidemiol Infect. (2016) 144:671-85. doi: 10.1017/S0950268815002575

46. Franc KA, Krecek RC, Häsler BN, Arenas-Gamboa AM. Brucellosis remains a neglected disease in the developing world: a call for interdisciplinary action. BMC Public Health. (2018) 18:125. doi: 10.1186/s12889-017-5016-y

47. Singha BB, Dhandb NK, Gilla JPS. Economic losses occurring due to brucellosis in Indian livestock populations. Prev Vet Med. (2015) 119:211-5. doi: $10.1016 /$ j.prevetmed.2015.03.013

48. Kreizinger Z, Foster JT, Rónai Z, Sulyok KM, Wehmann E, Jánosi $\mathrm{S}$, et al. Genetic relatedness of Brucella suis biovar 2 isolates from hares, wild boars and domestic pigs. Vet Microbiol. (2014) 27:492-8. doi: 10.1016/j.vetmic.2014.05.031

49. Pappas G, Papadimitriou P, Akritidis N, Christou L, Tsianos EV. The new global map of human brucellosis. Lancet Infect Dis. (2006) 6:91-9. doi: 10.1016/S1473-3099(06)70382-6

50. McDermott J, Grace D, Zinsstag J. Economics of brucellosis impact and control in low-income countries. Rev Sci Tech. (2013) 32:249-61. doi: 10.20506/rst.32.1.2197

51. Godfroid J, Käsbohrer A. Brucellosis in the European Union and Norway at the turn of the twenty-first century. Vet Microbiol. (2002) 90:135-45. doi: 10.1016/S0378-1135(02)00217-1

52. Davis DS, Elzer PH. Brucella vaccines in wildlife. Vet Microbiol. (2002) 90:533-44. doi: 10.1016/S0378-1135(02)00233-X

53. Yagupsky P, Baron EJ. Laboratory exposures to brucellae and implications for bioterrorism. Emerg Infect Dis. (2005) 11:1180-5. doi: 10.3201/eid1108.041197

54. Grégoire F, Mousset B, Hanrez D, Michaux C, Walravens K, Linden A. A serological and bacteriological survey of brucellosis in wild boar (Sus scrofa) in Belgium. BMC Vet Res. (2012) 8:80. doi: 10.1186/1746-614 8-8-80

55. Ferris RA, Schoenbaum MA, Crawford RP. Comparison of serologic tests and bacteriologic culture for detection of brucellosis in swine from naturally infected herds. J Am Vet Med Assoc. (1995) 207:1332-3.

56. Godfroid J, Michel P, Uytterhaegen L, Smedt CD, Rasseneur F, Saegerman C, et al. Brucella suis biotype 2 infection of wild boars (Sus scrofa) in Belgium. Ann Med Vet. (1994) 138:263-8.

57. Cvetnic Z, Mitak M, Ocepek M, Lojkic M, Terzić S, Jemersic L, et al. Wild boars (Sus scrofa) as reservoirs of Brucella suis biovar 2 in Croatia. Acta vet Hung. (2003) 51:465-73. doi: 10.1556/avet.51.2003.4.4

58. Leuenberger R, Boujon P, Thür B, Miserez R, Garin-Bastuji B, Rüfenacht J, et al. Prevalence of classical swine fever, Aujeszky's disease and brucellosis in a population of wild boar in Switzerland. Vet Rec. (2007) 160:362-8. doi: $10.1136 /$ vr.160.11.362

59. Closa-Sebastià F, Casas-Díaz E, Cuenca R, Lavín S, Mentaberre G, Marco I. Brucella species antibodies and isolation in wild boar in north-east Spain. Vet Rec. (2010) 167:826-8. doi: 10.1136/vr.c5927

60. Muñoz PM, Boadella M, Arnal M, de Miguel MJ, Revilla M, Martínez D, et al. Spatial distribution and risk factors of brucellosis in Iberian wild ungulates. BMC Infect Dis. (2010) 10:46. doi: 10.1186/1471-2334-10-46

61. Wu N, Abril C, Hinić V, Brodard I, Thür B, Fattebert J, et al. Free-ranging wild boar: a disease threat to domestic pigs in Switzerland? J Wildl Dis. (2011) 47:868-79. doi: 10.7589/0090-3558-47.4.868

62. Garin-Bastuji B, Hars J, Calvez D, Thiébaud M, Artois M. Brucellose du porc domestique et du sanglier sauvage due ?? Brucella suis biovar 2 en France. Epidémiol Santé Anim. (2000) 38:1-5.

63. Hubálek Z, Treml F, Juricová Z, Hunady M, Halouzka J, Janík V, et al. Serological survey of the wild boar (Sus scrofa) for tularaemia and brucellosis in South Moravia, Czech Republic. Vet Med. (2002) 47:3-60. doi: 10.17221/5805-VETMED

64. Cvetnic Z, Toncic J, Spicic S, Lojkic M, Terzic S, Jemersic L, et al. Brucellosis in wild boar (Sus scrofa) in the republic of Croatia. Vet Med. (2004) 49:115-22. doi: 10.17221/5684-VETMED

65. Godfroid J, Cloeckaert A, Liautard JP, Kohler S, Fretin D, Walravens K, et al. From the discovery of the Malta fever's agent to the discovery of a marine mammal reservoir, brucellosis has continuously been a re-emerging zoonosis. Vet Res. (2005) 36:313-26. doi: 10.1051/vetres:2005003

66. Megersa B, Biffa D, Niguse F, Rufael T, Asmare K, Skjerve E. Cattle brucellosis in traditional livestock husbandry practice in Southern and Eastern Ethiopia, and its zoonotic implication. Acta Vet Scand. (2011) 53:24. doi: 10.1186/1751-0147-53-24

67. Terefe Y, Girma S, Mekonnen N, Asrade B. Brucellosis and associated risk factors in dairy cattle of eastern Ethiopia. Trop Anim Health Prod. (2017) 49:599-606. doi: 10.1007/s11250-017-1242-7

68. Bugeza J, Muwonge A, Munyeme M, Lasuba P, Godfroid J, Kankya C. Seroprevalence of bovine brucellosis and associated risk factors in Nakasongola district, Uganda. Trop Anim Health Prod. (2019) 51:2073-6. doi: 10.1007/s11250-018-1631-6

69. Omer MK, Asfaw T, Skjerue E, Tekleghiorgis T, Woldehiwot T. Risk factors for Brucella spp. infection in dairy cattle farms in Asmara, State of Eritrea. Pre Vet Med. (2000) 46:257-65. doi: 10.1016/s0167-5877(00)00152-5

70. Ibrahim N, Belihu K, Lobago F, Bekana M. Sero-prevalence of bovine brucellosis and its risk factors in Jimma zone of Oromia Region, South-western Ethiopia. Trop Anim Health Prod. (2010) 42:35-40. doi: 10.1007/s11250-009-9382-z

71. Abedi A, Hashempour-Baltork F, Alizadeh AM, Beikzadeh S, Hosseini H, Bashiry M, et al. The prevalence of Brucella spp. In dairy products in the middle east region: a systematic review and meta-analysis. Acta Trop. (2019) 202:105241. doi: 10.1016/j.actatropica.2019.105241

72. Goonaratna C. Brucellosis in humans and animals. Ceylon Med J. (2009) 52:66. doi: $10.4038 / \mathrm{cmj} . v 52 \mathrm{i} 2.1028$

73. Meirelles-Bartoli RB, Mathias LA, Samartino LE. Brucellosis due to Brucella suis in a swine herd associated with a human clinical case in the State of São Paulo, Brazil. Trop Anim Health Prod. (2012) 44:1575-9. doi: 10.1007/s11250-012-0108-2

74. Barlozzari G, Franco A, Macrì G, Lorenzetti S, Maggiori F, Dottarelli S, et al. First report of Brucella suis biovar 2 in a semi free-range pig farm, Italy. Vet Ital. (2015) 51:151-4. doi: 10.12834/VetIt.50.3384.1

75. Pilo C, Tedde MT, Orrù G, Addis G, Liciardi M. Brucella suis infection in domestic pigs in Sardinia (Italy). Epidemiol Infect. (2015) 143:2170-7. doi: 10.1017/S0950268814003513

76. Ferreira AC, Tenreiro R, de Sá MIC, Dias R. Complete genome sequences of three iberian Brucella suis Biovar 2 strains isolated from wild boars. Genome Announc. (2014) 2:e00618-4. doi: 10.1128/genomeA. 00618-14

77. Melzer F, Lohse R, Nieper H, Liebert M, Sachse K. A serological study on brucellosis in wild boars in Germany. Eur J Wildlife Res. (2006) 53:153-7. doi: 10.1007/s10344-006-0072-0

78. Knight-Jones TJ, Mylrea GE, Kahn S. Animal production food safety: priority pathogens for standard setting by the world organisation for animal health Rev Sci Tech. (2010) 29:523-35. doi: 10.20506/rst.29.3.1994

79. Ruiz-Fons F, Vidal D, Höfle U, Vicente J, Gortázar C. Aujeszky's disease virus infection patterns in European wild boar. Vet Microbiol. (2007) 120:241-50. doi: 10.1016/j.vetmic.2006.11.003

80. Llario PF. Ecología del Jabalí en Doñana: Parámetros Reproductivose Impacto Ambiental. Cáceres: Univ of Extremadura (1996).

81. Tounkara K, Maiga S, Traoré A, Seck BM, Akakpo AJ. Epidemiology of bovine brucellosis in mali: serologic investigation and initial isolation of strains of Brucella abortus. Rev Sci Tech. (1994) 13:777-86. doi: 10.20506/rst.13.3.795

82. Jori F, Godfroid J, Michel AL, Potts AD, Jaumally MR, Sauzier J, et al. An assessment of zoonotic and production limiting pathogens in rusa deer (Cervus timorensis rusa) from Mauritius. Transbound Emerg Dis. (2014) 61(Suppl 1):31-42. doi: 10.1111/tbed.12206

Conflict of Interest: The authors declare that the research was conducted in the absence of any commercial or financial relationships that could be construed as a potential conflict of interest.

Copyright (c) 2021 Gong, Sun, Yang, Zhao, Wang, Li, Ge, Chen, Shi, Leng, Zong and $\mathrm{Du}$. This is an open-access article distributed under the terms of the Creative Commons Attribution License (CC BY). The use, distribution or reproduction in other forums is permitted, provided the original author(s) and the copyright owner(s) are credited and that the original publication in this journal is cited, in accordance with accepted academic practice. No use, distribution or reproduction is permitted which does not comply with these terms. 